The ACER pollen and charcoal database: a global resource to document vegetation and fire response to abrupt climate changes during the last glacial period

ACER Project Members*: M.F. Sánchez Goñi ${ }^{1,2}$, S. Desprat ${ }^{1,2}$, A.-L. Daniau ${ }^{3}$, F. Bassinot ${ }^{4}$, J.M. PolancoMartínez $^{2,5}$, S.P. Harrison ${ }^{6,7}$, and ACER contributors

\title{
Supplementary Information
}

Taxa defining the pollen percentages of the main biomes in South Africa, Kenya, Australia and New Zealand not included in the QSR special issue (Sánchez Goñi and Harrison, 2010).

\section{Mfabeni Peatland (South Africa)}

Temperate savannah: Anacardiaceae, Ericaceae, Euphorbiaceae, Fabaceae, Fabaceae (Acacia), Proteaceae.

Warm-temperate mixed forest: Apocynaceae, Celastraceae, Combretaceae, Cyanthaceae, Erythroxylaceae, Flacourtiaceae, Moraceae, Myricaceae, Myrtaceae, Podocarpaceae, Rosaceae, Rubiaceae.

\section{Rumuiku Swamp (Kenya)}

Temperate forest : Ilex, Celtis, Lannea, Malvaceae, Rubiaceae, Rhus, Rubus, Stoebe, Merrema, Tiliaceae, Oenostachys, Commelina, Abutilon, Clematis, Cissampelos, Cardamine, Amaranthaceae/Chenopodiaceae, Acanthaceae, Cleome, Cocculus, Plectranthus, Cucurbitaceae, Caryophyllaceae, Cuscuta, Kedrostis, Ranunculus, Gynandropsis, Iridaceae, Hygrophila, Heliotropium, Leucas, Lamiaceae, Liliaceae, Fabaceae, Trema, Valeriana, Ipomoea, Solanum, Urticaceae, Ericaceae, Asteraceae, Brassicaceae, Apiaceae, Artemisia, Poaceae

Warm temperate forest: Dombeya, Myrica, Nuxia, Olea, Moraceae, Podocarpus, Polyscias, Protea, Schefflera, Hagenia, Alchornea, Ilex, Macaranga, Afrocrania, Celtis, Croton, Juniperus, Rubiaceae, Rapanea, Lasianthus, Syzygium, Capparidaceae, Allophylus, Apodytes, Hypericum, Acalypha, Albizia, Antidesma, Acacia, Bosquea, Canthium, Cliffortia, Neoboutonia, Clausena, Combretum, Clerodendron, Cordia, Drypetes, Dracaena, Phyllanathus, Elatine, Ekebergia, Euclea, Faurea, Gunnera, Gnidia, Ziziphus, Lannea, Malvaceae, Maesa, Phyllanthus, Prunus, Ruelia, Rutaceae, Rubiaceae, Rhus, Rubus, Sapindaceae, Sapotaceae, Tapinanthus, Merremia, Tiliaceae, Oenostachys, Commelina, Abutilon, Clematis, Cissampelos, Cardamine, Amaranthaceae/Chenopodiaceae, Ricinus, Acanthaceae, Cleome, Cocculus, Plectranthus, Cucurbitaceae, Caryophyllaceae, Cuscuta, Chlorophytum, Corchorus, Kohautia, Vernonia, Pavetta, Anthospermum, Ranunculus, Galium, Gynandropsis, Iridaceae, Hyptis, Hygrophila, Leucas, Lamiaceae, Hypoestes, Fabaceae, Trema, Valeriana, Ipomoea, Indigofera, Solanum, Urticaceae, Ericaceae, Asteraceae, Brassicaceae, Apiaceae, Artemisia, Poaceae. 
Tropical forest: Dombeya, Myrica, Nuxia, Olea, Moraceae, Podocarpus, Polyscias, Protea, Schefflera, Hagenia, Alchornea, Ilex, Macaranga, Afrocrania, Celtis, Croton, Juniperus, Rubiaceae, Rapanea, Lasianthus, Syzygium, Capparidaceae, Allophylus, Apodytes, Hypericum, Acalypha, Albizia, Antidesma, Acacia, Bosquea, Canthium, Cliffortia, Neoboutonia, Clausena, Combretum, Clerodendron, Cordia, Drypetes, Dracaena, Phyllanathus, Elatine, Ekebergia, Euclea, Faurea, Gunnera, Gnidia, Ziziphus, Lannea, Malvaceae, Maesa, Phyllanthus, Prunus, Ruelia, Rutaceae, Rubiaceae, Rhus, Rubus, Sapindaceae, Sapotaceae, Tapinanthus, Merremia, Tiliaceae, Oenostachys, Commelina, Abutilon, Clematis, Cissampelos, Cardamine, Amaranthaceae/Chenopodiaceae, Ricinus, Acanthaceae, Cleome, Cocculus, Plectranthus, Cucurbitaceae, Caryophyllaceae, Cuscuta, Chlorophytum, Corchorus, Kohautia, Vernonia, Pavetta, Anthospermum, Galium, Gynandropsis, Iridaceae, Hyptis, Hygrophila, Lamiaceae, Hypoestes, Fabaceae, Ipomoea, Indigofera, Ericaceae, Asteraceae, Brassicaceae, Apiaceae, Poaceae.

\section{Caledonia Fen and Wagoom (Australia)}

Warm temperate forest: Podocarpus, Phylloclades.

Savannah: Eucalyptus, Casuarina, Poaceae, Asteraceae, Apiaceae, Banksia, Pomaderris, Acacia, Dodonaea, Plantago.

\section{Kohuora (New Zealand)}

Warm temperate forest: Agathis, Alectryon, Ascarina, Dacrydium, Dacrycarpus, Dodonaea, Elaeocarpus, Griselinia, Knightia, Laurelia, Leucopogon fasciculatus, Libocedrus plumosa, Metrosideros, Metrosideros excelesa type, Neomyrtus, Nestegis, Phyllocladus trichomanoides, Plagianthus, Podocarpus, Prumnopitys taxifolia, Prumnopitys ferruginea, Pseudopanax, Weinmannia, Cyathea dealbata type, Cyathea smithii type.

Temperate forest: Fuscospora, Griselinia, Halocarpus bidwillii, Hoheria, Lagarostrobos, Lepidothamnus, Libocedrus bidwillii, Muehlenbeckia, Nothofagus menziesii, Phyllocladus alpinus, Plagianthus, Podocarpus, Quintinia.

Table S1 - List of the applied and selected age models for the sites included in the ACER database.

LI: Liner interpolation; LR: Linear regression; PR2: Polynomial regression-order 2; PR3: Polynomial regression-order 3; PR4: Polynomial regression-order 4; CS: Cubic spline; SSO.3: Smooth spline (smoothing 0.3); SSO.6: Smooth spline (smoothing 0.6); LW0.75: Locally weighted spline (smoothing 0.75). Green cells indicate the selected age model.

No new age model for the following sites: Bear Lake; Lago Grande di Monticchio (too many major inversions in the 14C dates); Okarito Pakihi (lack of dating information); EW9504-17PC; F2-92-P29; ODP 1234; Wonderkrater (Borehole 3); Huiñamarca (Lake Titicaca, lacking dating uncertainties for tephra and $\mathrm{U} / \mathrm{Th}$ dates). 


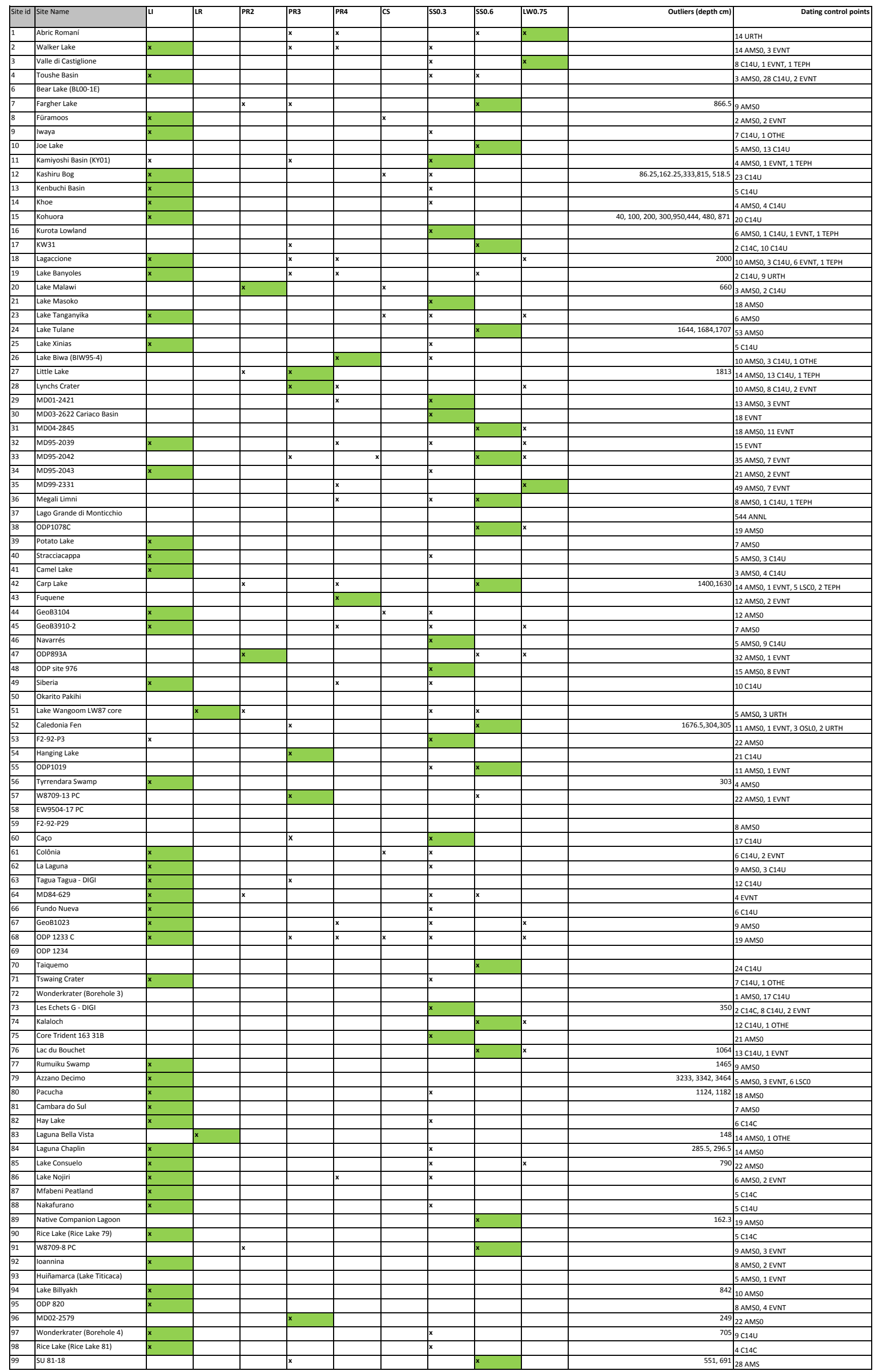

